\title{
CINTIGRAFIA PULMONAR POR PERFUSION EN NIÑOS CON DAÑO PULMONAR CRONICO: RESULTADOS PRELIMINARES DE LA APLICACION DE LA TECNICA SPECT
}

\author{
Drs. Gabriel Lobo $S^{(1)}$, David Ladrón de Guevara $H^{(1)}$, María Angélica Muñoz $G^{(1)}$, Fernando Araya $A^{(2)}$, \\ Claudia Coll $\boldsymbol{C}^{(1)}$, Gilda Donoso $\boldsymbol{R}^{(1)}$, César Jiménez $\boldsymbol{J}^{*(1)}$, Andrés Pérez $\boldsymbol{R}^{*(1)}$.
}

1. Departamento de Medicina Nuclear, Hospital San Juan de Dios.

2. Departamento de Pediatría, Hospital San Juan de Dios.

* Tecnólogo Médico

\begin{abstract}
The aim was to describe lung perfusion SPECT (LPS) findings in children with chronic pulmonary damage. Material and Method: We revised 106 LPS of children (age:3.9 $\pm 3.3 \mathrm{yr}$ ) with chronic pulmonary damage, performed with a two headed gammacamera. The most common clinical diagnosis were adenovirus bronchopneumonia sequelae $(A D V)(29 \%)$, unknown origin chronic pulmonary damage (UOPD) (21\%), bronchopulmonary dysplasia $(B P D)(15 \%)$, and chronic obstructed bronchitis $(C O B)(14 \%)$. Severity of pulmonary alterations were evaluated using a Score, which considered extent and type of lung involvement and differential pulmonary perfusion. Pattern of involvement and Score were correlated with diagnosis and gender. Results: Ninety one percent (96/106) of LPS were abnormal (62\% boys). Fifty two (54\%) showed bilateral alterations, being this finding ( $p: 0.019)$ more frequent in boys. ADV group showed mainly a mixed bilateral pattern (35\%), UOPD a focal unilateral pattern (32\%), COB a diffuse unilateral pattern (33\%), and BPD a normal pattern (25\%). Miscellaneous and UOPD showed the highest score values, and BPD and $C O B$ the lowest ones. Conclusion: In this population, LPS is abnormal in high proportion, with some differential characteristics according gender and original diagnosis.
\end{abstract}

Lobo G. y cols. Cintigrafía pulmonar por perfusión en niños con daño pulmonar crónico: Resultados preliminares de la aplicación de la tecnica SPECT. Rev Chil Radiol 2003; 9: 117-122.

Correspondencia: Dr. Gabriel Lobo S.

Unidad de Medicina Nuclear, CDT Hospital San Juan de Dios.

Portales 3239, Santiago Centro

Fono: 4506869 - Fonofax: 6825314

globo@machi.med.uchile.cl
Key words: Chronic pulmonary damage, Children, Lung perfusion SPECT, Pulmonary scintigraphy.

Resumen: El objetivo fue describir los hallazgos del SPECT de perfusión pulmonar (SPP) en niños con daño pulmonar crónico. Material y Método: Se

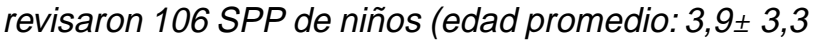
años) con daño pulmonar crónico realizados con gammacámara doble cabezal. Los diagnósticos más frecuentes fueron secuela de BNM por adenovirus (ADV) (29\%), daño pulmonar crónico de etiología desconocida (DPED) (21\%), displasia broncopulmonar (DBP) (15\%), y síndrome bronquial obstructivo (SBO) (14\%). El grado de alteración se evaluó utilizando un Score, que consideró extensión y tipo de compromiso y perfusión pulmonar diferencial. El tipo de compromiso y Score fueron correlacionados con diagnóstico y sexo. Resultados: El 91\% (96/106) de los estudios fueron anormales (62\% varones), 52 (54\%) con alteraciones bilaterales, más frecuentes en varones $(p: 0,019)$. El grupo ADV mostró principalmente (35\%) un patrón mixto bilateral, el DPED uno unilateral focal (32\%), el $S B O$ uno unilateral difuso (33\%), y la DBP uno normal (25\%). Misceláneas y DPED presentaron los Score más altos (alteraciones severas), y DBP y SBO los más bajos. Conclusión: En esta población el SPP se altera en una alta proporción con algunas características diferenciales según sexo y etiología.

Palabras clave: Cintigrama pulmonar, Daño pulmonar crónico, Niños, SPECT perfusión pulmonar

\section{Introducción}

El daño pulmonar crónico persistente y progresivo en niños parece ser un factor predisponente a la disfunción pulmonar y sintomatología broncopulmonar crónica en el adulto. En nuestro medio la causa más frecuente de dicha patología en niños son las 
infecciones respiratorias virales, particularmente por adenovirus.

En la literatura hay escasas referencias respecto a la aplicación de la cintigrafía pulmonar ${ }^{(1,2,3)}$ y específicamente de la técnica de SPECT (single photon emission computed tomography) en la evaluación de estos pacientes ${ }^{(4)}$. Esto pese a que permite una evaluación morfofuncional broncopulmonar que provee información funcional y estructural respecto al lecho vascular pulmonar, y además no requiere de gran colaboración de los pacientes, lo que facilita su uso en niños de menor edad. De esta manera la cintigrafía pulmonar podría ser útil en la evaluación del grado de compromiso estructural y funcional residual en niños con daño pulmonar crónico, con proyecciones clínicas en la predicción del pronóstico y seguimiento evolutivo.

La técnica SPECT aplicada a la cintigrafía pulmonar de perfusión ha demostrado ser superior a la planar para el diagnóstico de tromboembolismo pulmonar ${ }^{(5)}$ con una mayor sensibilidad y especificidad en la pesquisa de lesiones, mejor localización de éstas y mayor facilidad de correlación con otras técnicas tomográficas actualmente en uso en pacientes con patología broncopulmonar ${ }^{(4,6)}$. Por otra parte, los avances tecnológicos en la metodología SPECT permiten una mayor rapidez en la realización del examen mediante el uso de cámaras con múltiples cabezales, con mejores métodos de procesamiento obteniéndose imágenes de mayor calidad, y nuevos programas para detección de artefactos que conlleva una menor frecuencia de resultados falsos positivos por movimiento del paciente y otras dificultades inherentes a la técnica tomográfica.

El objetivo de este trabajo fue evaluar preliminarmente los hallazgos del SPECT pulmonar de perfusión en niños con daño pulmonar crónico, describiendo las anomalías encontradas y proponer un método para graduar el daño pulmonar basado en dicha técnica.

\section{Pacientes y método}

Se estudiaron prospectivamente 106 niños (edad: $3,9 \pm 3,8$ años, rango: 1 mes a 15 años), 62,3\% varones, controlados ambulatoriamente en el Departamento de Broncopulmonar del Servicio de Pediatría del Hospital San Juan de Dios, por daño pulmonar crónico. Estos niños fueron enviados en períodos intercríticos sin evidencias de reagudización a la unidad de Medicina Nuclear de dicho Hospital, para realizarse una cintigrafía pulmonar por perfusión, entre Octubre 2000 y Enero 2002. Los principales diagnósticos clínicos, frecuencia y distribución por edad y sexo se muestran en la Tabla I. Los diagnósticos más frecuentes fueron: secuela de bronconeumonía por adenovirus (ADV) (29\%), daño pulmonar crónico de etiología desconocida (DPED) (21\%), displasia broncopulmonar (DBP) (15\%), y síndrome bronquial obstructivo (SBO) (14\%). El grupo Misceláneas (MISC) correspondió principalmente a pacientes referidos por daño encefálico-Síndrome Down, reflujo gastroesofágico severo, infección por $\mathrm{VIH}$, malformaciones y fibrosis quística.

La técnica SPECT se realizó en una gammacámara SMV DST XLi doble cabezal 1999, con colimador de baja energía y alta resolución, adquisición en matriz $128 \times 128,32$ proyecciones por cabezal, de $15 \mathrm{~s}$ cada una, con una duración total del examen menor de $10 \mathrm{~min}$. Se utilizó macroagregados de albúmina-Tc $\mathrm{c}^{99 m}$ (MAA-Tc ${ }^{99 m}$ ) ajustando la dosis según edad, administrado por vía e.v. en posición decúbito dorsal. Los pacientes no colaboradores fueron inmovilizados con saco al vacío. Se adquirieron además imágenes planares frontal y posterior para cálculo de la perfusión pulmonar relativa (PPR) basada en el promedio geométrico. Las imágenes fueron reconstruidas siguiendo un método iterativo con 2 iteraciones y filtrado posterior (Butterwotrh order 5 , cutoff 0,25 ). Previamente se analizaron las imágenes planares en modo "cine" y los sinogramas para detectar movimiento.

Tabla I. Diagnósticos de referencia de la población estudiada según sexo y grupo etario

\begin{tabular}{|c|c|c|c|c|c|c|c|c|}
\hline & \multicolumn{4}{|c|}{ Hombres } & \multicolumn{4}{|c|}{ Mujeres } \\
\hline & 0-2 años & 2-5 años & >5 años & Total & 0-2 años & 2-5 años & >5 años & Total \\
\hline ADV $(n=31)$ & 10 & 7 & 3 & 20 & 8 & 1 & 2 & 11 \\
\hline DPED (n=22) & 2 & 4 & 4 & 10 & 3 & 4 & 5 & 12 \\
\hline $\operatorname{DBP}(n=16)$ & 6 & 4 & 0 & 10 & 4 & 2 & 0 & 6 \\
\hline SBO $(n=15)$ & 3 & 4 & 4 & 11 & 2 & 0 & 2 & 4 \\
\hline $\operatorname{VRS}(\mathrm{n}=7)$ & 7 & 0 & 0 & 7 & 0 & 0 & 0 & 0 \\
\hline MISC $(n=15)$ & 5 & 1 & 2 & 8 & 2 & 1 & 4 & 7 \\
\hline TOTAL $(n=106)$ & 33 & 20 & 13 & 66 & 19 & 8 & 13 & 40 \\
\hline
\end{tabular}

ADV: Secuela de bronconeumonía por Adenovirus, DPED: daño pulmonar crónico de etiología desconocida, DBP: displasia broncopulmonar, SBO: síndrome bronquial obstructivo, VRS: secuela de bronconeumonía por virus respiratorio sincicial, MISC: grupo misceláneo. 
La interpretación del estudio fue realizada por dos observadores experimentados, en las imágenes 3D y cortes tomográficos.

Se reconoció un patrón cintigráfico normal y tres anormales (lesiones focales, lesiones difusas o lesiones mixtas).

Se calculó un score del grado de alteración de la cintigrafía basado en la existencia de alteraciones focales y/o difusas y en la PPR de acuerdo al criterio presentado en la Tabla II. El score 0 correspondió a estudios normales (Figura 1), el score 1 a alteraciones leves (Figura 2), el 2-3 a alteraciones moderadas (Figura 3), y los valores $>3$ a alteraciones severas (Figura 4).

El score obtenido y el patrón cintigráfico se correlacionó con el sexo y diagnóstico etiológico de los pacientes. Para el análisis estadístico se utilizó prueba de Chi cuadrado.

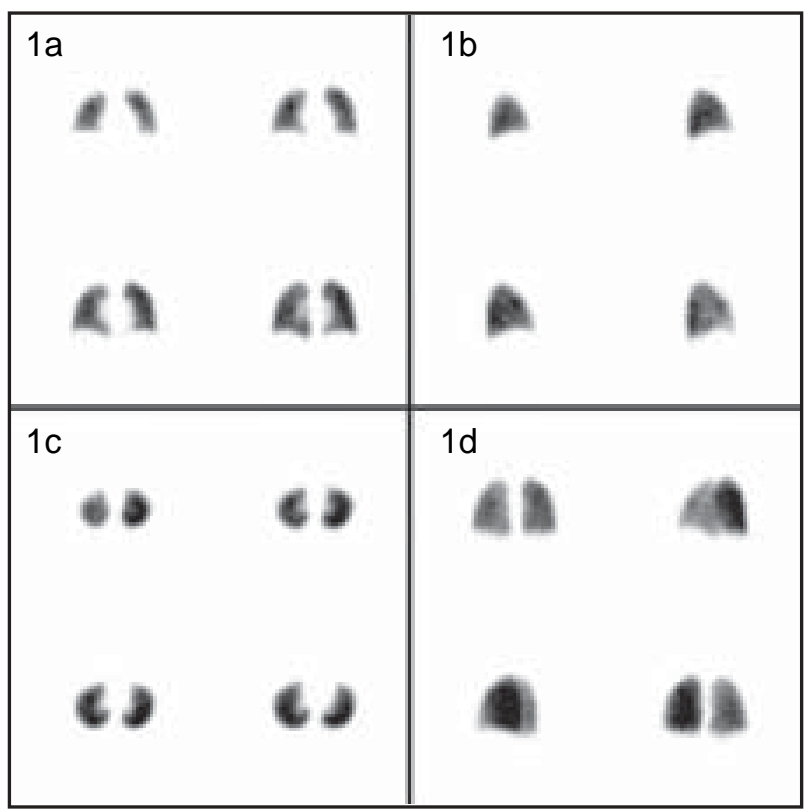

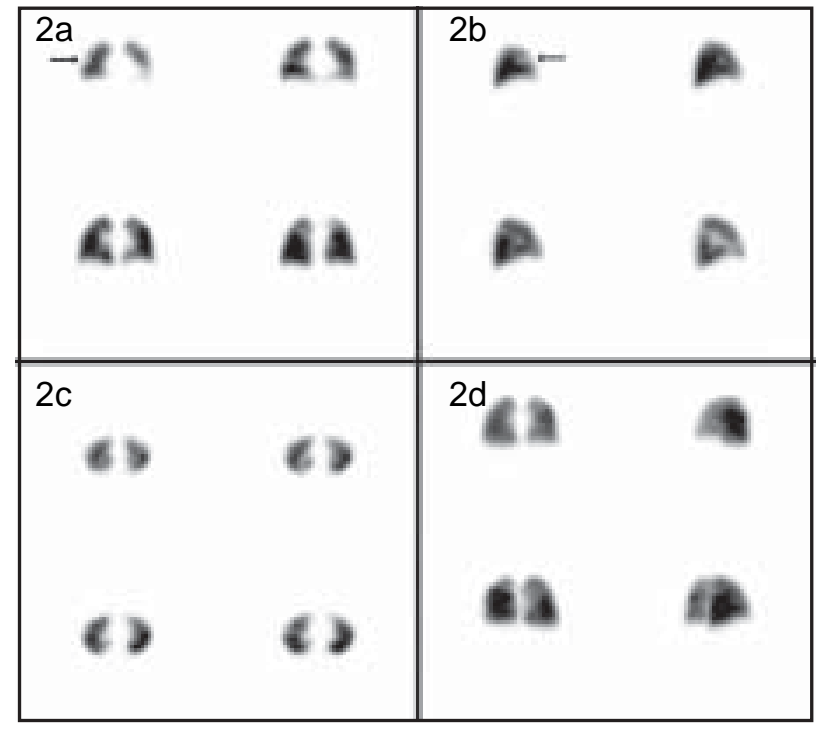

Figura 2. SPECT de perfusión Pulmonar con score leve: se observa defecto de perfusión focal en segmento posterior del lóbulo superior derecho (1 punto), sin alteraciones difusas, con función pulmonar diferencial normal (derecho:58\%, izquierdo:42\%). Total score 1 punto. A) Imágenes en cortes coronales: defecto en pulmón derecho con flecha negra, B) Imágenes en cortes sagitales, C) Imágenes en cortes axiales, D) Imágenes en 3 dimensiones.

Figura 1. SPECT de Perfusión Pulmonar con distribución Normal del radiofármaco.

A) Imágenes en cortes coronales, $B$ ) Imágenes en cortes sagitales, C) Imágenes en cortes axiales, D) Imágenes en 3 dimensiones.

Tabla II. Criterios cintigráficos para el cálculo de score de severidad

A) Indice de compromiso focal (máximo 3 puntos)

Compromiso unilateral leve o moderado $(<0=3$ segmentos $)$

Compromiso unilateral severo ( $>3$ segmentos)

Compromiso bilateral leve o moderado ( 2 a 6 segmentos totales)

Compromiso bilateral severo (> 6 segmentos totales)

B) Indice de compromiso difuso (máximo 3 puntos)

Compromiso difuso unilateral

Compromiso difuso bilateral

Compromiso difuso unilateral asociado a lesión(es) focal(es)

Compromiso difuso bilateral asociado a lesión(es) focal(es)

C) Indice de compromiso de PPR (máximo 2 puntos)

PPR 35 a $40 \%$

PPR $<35 \%$

Puntaje

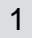

2

2

3

1

2

2

3

1

2

Score: Suma $A+B+C$ (máximo: 8 puntos)

PPR: Perfusión pulmonar relativa. 


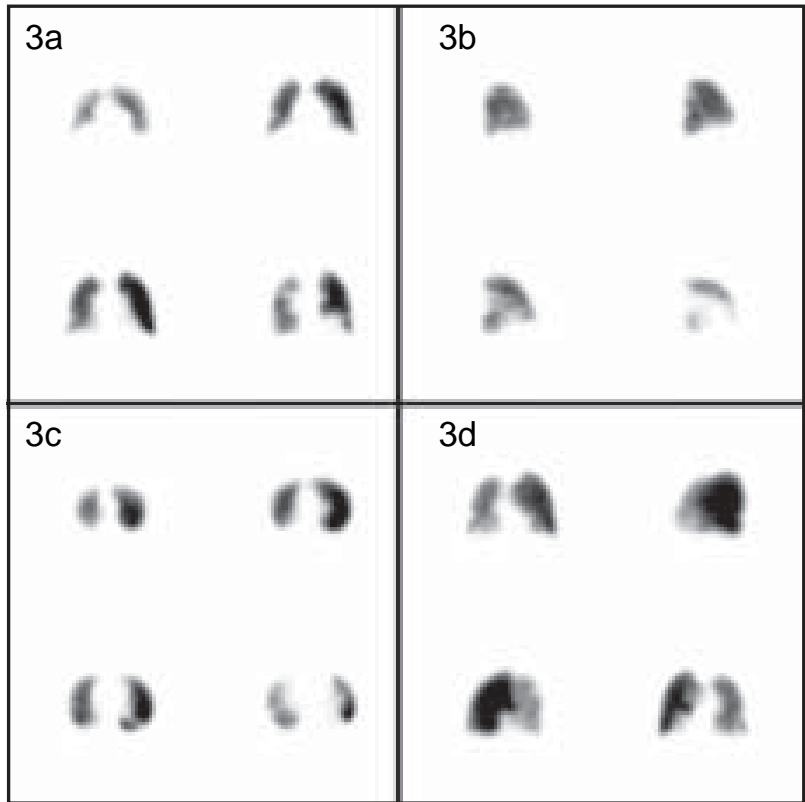

Figura 3. SPECT de perfusión Pulmonar con score moderado: se observa defectos de perfusión focales segmentarios en lóbulos superior e inferior derechos y del lóbulo inferior izquierdos (2 puntos), sin alteraciones difusas, con función pulmonar diferencial anormal (derecho:39\%, izquierdo: 61\%). Total score 3 puntos.

A) Imágenes en cortes coronales, B) Imágenes en cortes sagitales, C) Imágenes en cortes axiales, D) Imágenes en 3 dimensiones.

\section{Resultados}

No se rechazaron exámenes por movimiento de los pacientes u otros artefactos.

Del total de SPECT pulmonares, 90,6\% (96/106) fueron anormales (62\% varones), como se muestra en la Tabla III. El $54,2 \%$ de los estudios anormales (52/96) mostraron alteraciones bilaterales, las que fueron significativamente más frecuentes en varones que en mujeres $(56,1 \%$ vs $37,5 \%$, respectivamente)(p:0,019). El grupo que presentó alteraciones unilaterales tuvo frecuencia similar de compromiso del pulmón izquierdo y derecho.

Del total de estudios, $16,9 \%$ presentó patrón de

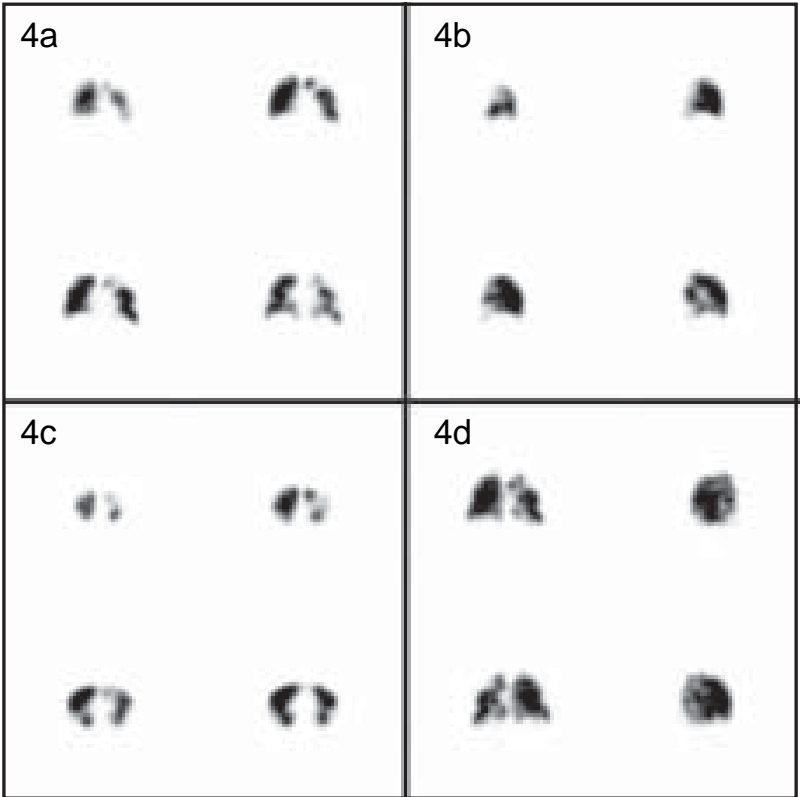

Figura 4. SPECT de perfusión Pulmonar con score severo: se observa múltiples defectos de perfusión focales bilaterales (3 puntos), con alteración difusa izquierda (2 puntos), con función pulmonar diferencial normal (derecho: 59\%, izquierdo:41\%). Total score 5 puntos.

A) Imágenes en cortes coronales; B)Imágenes en cortes sagitales; C)Imágenes en cortes axiales; D) Imágenes en 3 dimensiones.

compromiso difuso, 42,4\% mostró lesiones focales, y $31,1 \%$ tuvo un patrón mixto difuso-focal (Tabla III).

La distribución de los parámetros cintigráficos de acuerdo al diagnóstico se presentan en la Tabla III, donde se aprecia que el daño por adenovirus mostró con mayor frecuencia $(35,5 \%)$ un patrón mixto bilateral, el DPED un patrón unilateral focal $(31,8 \%)$, el SBO un patrón unilateral difuso (33,3\%), y la DBP uno normal (25\%). La localización de las alteraciones focales se presentan en la Figura 5 , siendo la localizaciones más frecuentes el lóbulo inferior derecho, lóbulo inferior izquierdo y lóbulo superior derecho.

Tabla III. Patrón cintigráfico según diagnóstico de referencia en pacientes en estudio por daño pulmonar crónico.

\begin{tabular}{|c|c|c|c|c|c|c|c|c|c|c|c|}
\hline \multirow{2}{*}{\multicolumn{2}{|c|}{ NORMALES (\%) }} & \multicolumn{10}{|c|}{ ANORMALES (\%) } \\
\hline & & Unilat & $\begin{array}{l}\text { Difus } \\
\text { Bilat }\end{array}$ & Total & Unilat & $\begin{array}{l}\text { Focal } \\
\text { Bilat }\end{array}$ & Total & Unilat & $\begin{array}{l}\text { Mixto } \\
\text { Bilat }\end{array}$ & Total & TOTAL \\
\hline ADV $(n=31)$ & 3,2 & 6,4 & 3,2 & 9,6 & 12,9 & 25,8 & 38,7 & 12,9 & 35,5 & 48,4 & 100 \\
\hline DPED $(n=22)$ & 9,0 & 9,0 & 4,5 & 13,5 & 31,8 & 18,1 & 49,9 & 9,0 & 18,1 & 27,1 & 100 \\
\hline $\operatorname{DBP}(n=16)$ & 25,0 & 18,8 & 12,5 & 31,3 & 6,2 & 18,8 & 25,0 & 6,2 & 12,5 & 18,7 & 100 \\
\hline SBO $(n=15)$ & 13,3 & 33,3 & 6,6 & 39,9 & 13,3 & 20,0 & 33,3 & 0 & 13,3 & 13,3 & 100 \\
\hline VRS $(n=7)$ & 14,3 & 0 & 0 & 0 & 14,3 & 28,6 & 42,9 & 28,6 & 14,3 & 42,9 & 100 \\
\hline MISC $(n=15)$ & 0 & 6,6 & 0 & 6,6 & 40,0 & 26,6 & 66,6 & 6,6 & 20,0 & 26,6 & 100 \\
\hline TOTAL $(n=106)$ & 9,4 & 12,2 & 4,7 & 16,9 & 19,8 & 22,6 & 42,4 & 9,4 & 21,7 & 31,1 & 100 \\
\hline
\end{tabular}

ADV: Secuela de bronconeumonía por Adenovirus, DPED: daño pulmonar crónico de etiología desconocida, DBP: displasia broncopulmonar, SBO: síndrome bronquial obstructivo, VRS: secuela de bronconeumonía por virus respiratorio sincicial, MISC: grupo misceláneo. 


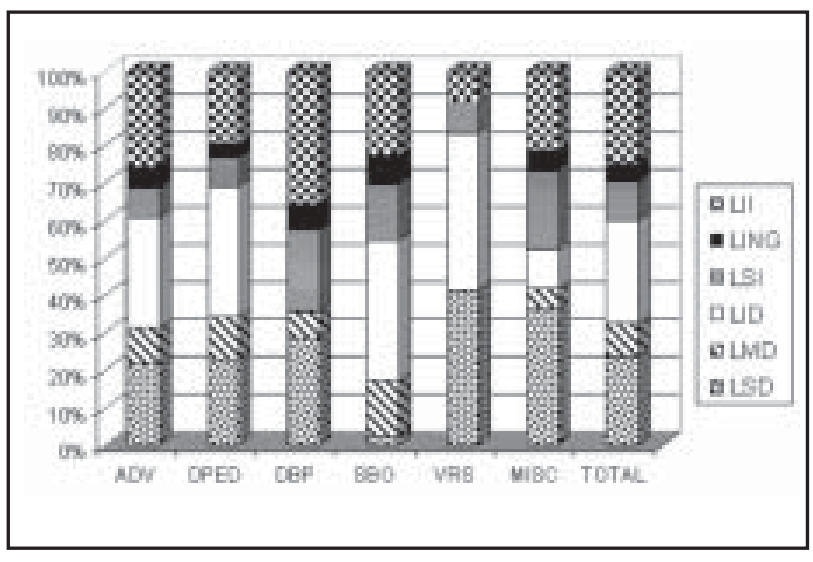

Figura 5. Frecuencia de localización de las alteraciones focales según patología y en el grupo de pacientes con SPECT alterado.

LII: Lóbulo inferior izquierdo, LING: língula, LSI: lóbulo superior izquierdo, LID: lóbulo inferior derecho, LMD: lóbulo medio derecho, lóbulo superior derecho. Resto abreviaturas como en la tabla I.

La distribución global y por patología de las alteraciones del score cuantitativo se muestra en la Figura 6. Las patologías que presentan score más alterado (>4) fueron el grupo MISC y ADV. Los grupos con mayor número de score normales fueron DBP y $\mathrm{SBO}$, los que no presentaron scores $>4$ (alteraciones severas).

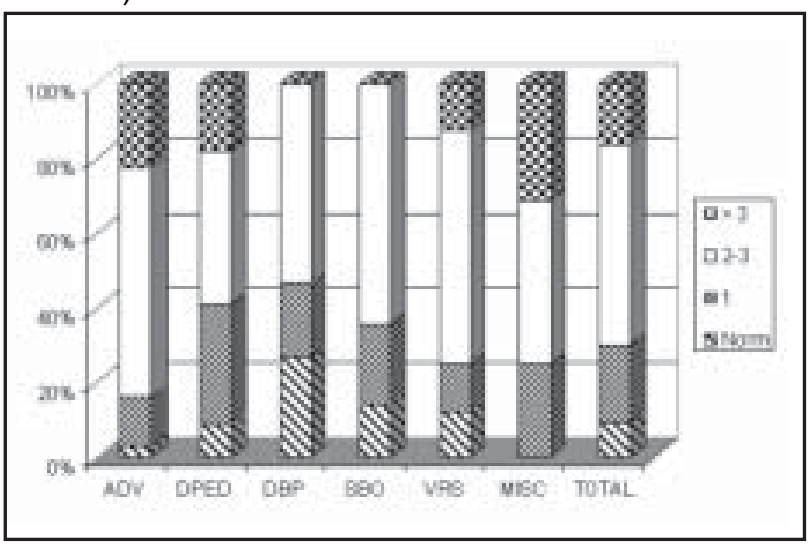

Figura 6. Frecuencia del score de daño pulmonar según patología y en el grupo total de pacientes estudiados. Abreviaturas como en las tabla I.

\section{Discusión}

Este trabajo describe algunas de las características de las alteraciones del SPECT pulmonar de perfusión en niños portadores de daño pulmonar crónico en período intercrítico y propone una metodología para evaluar semicuantitativamente la intensidad de la alteración cintigráfica.

La técnica de SPECT resultó en nuestra experiencia de aplicación expedita en niños, con tiempos de examen de corta duración y estudios de excelente calidad. Las imágenes fueron de interpretación sencilla, especialmente al evaluar en conjunto los cortes tomográficos con las imágenes en 3D (cine), obteniéndose una impresión global de la anatomía pulmonar más cercana a la realidad que con las imágenes planares, especialmente en presencia de anomalías de la caja toráxica como escoliosis, entre otras.

La incidencia de anormalidad del examen fue muy elevada en la casuística en general, especialmente en varones, aún cuando la mayor parte de las alteraciones se clasificaron como leves o moderadas de acuerdo al score propuesto. Estos resultados están de acuerdo con lo esperado en pacientes ambulatorios que reconocidamente son portadores de daño pulmonar crónico en período intercrítico. La severidad de las lesiones secuelares de pacientes que sufrieron infección por ADV ha sido descrito en diversas series ${ }^{(7,8)}$. Esta característica se evidenció también en nuestra casuística, en que este grupo obtuvo los valores de score más altos.

El mayor interés de la cintigrafía de perfusión pulmonar probablemente está en la evaluación del grado de daño pulmonar, tanto estructural como funcional. La información provista por esta técnica es estructural en la medida que indica localización y cuantía del daño del lecho vascular pulmonar y es funcional por cuanto evalúa alteraciones de la perfusión funcional regional del pulmón. Las alteraciones de la cintigrafía pulmonar pueden ser parcialmente reversibles en cuadros agudos o pueden indicar una alteración morfofuncional establecida cuando el estudio se realiza alejado del cuadro agudo. Una manera de graduar la intensidad de las alteraciones cintigráficas es a través de un método semicuantitativo como el score propuesto que si bien tiene elementos subjetivos como la apreciación del grado de compromiso focal o difuso, tiene también elementos objetivos como la cuantificación de la perfusión pulmonar relativa. Otras modalidades de imágenes pulmonares también han sido analizadas utilizando diversos Scores para graduar el daño pulmonar crónico en niños como los Scores de dilatación bronquial (Bhalla modificado) ${ }^{(9)}$ o la extensión de mosaico de perfusión, que evalúa indirectamente la perfusión pulmonar (Score de Siegel) ${ }^{(10)}$, el cual tiene cierta semejanza con el score propuesto en este trabajo.

La existencia de una correlación entre el grado de daño detectado por un método de imagen en niños con daño por ADV y el compromiso de la función pulmonar ha sido demostrada en trabajos en que comparan los resultados de la TC con la espirometría(11), particularmente evaluando la extensión del daño bronquial y del mosaico de perfusión mediante los scores señalados en el párrafo precedente, obteniéndose una correlación con ambos, aunque mayor con el Score de Siegel ${ }^{(10)}$.

Con el uso del SPECT de perfusión pulmonar se podría esperar una correlación similar o mejor 
con parámetros funcionales, por cuanto se evalúa más específica y directamente la perfusión pulmonar.

Este trabajo constituye un precedente para investigaciones futuras que contemplen comparación de la cintigrafía pulmonar con otros estudios funcionales como saturación de oxígeno, espirometría o estructurales como TC, y el seguimiento evolutivo de los pacientes.

De acuerdo a nuestra experiencia con este grupo de pacientes, creemos que la técnica de SPECT es simple de implementar en niños, especialmente usando una cámara de dos o más cabezales. Los artefactos, cuando existen, son fácilmente detectables y la interpretación del examen también es ventajosa sobre las imágenes planares, tanto por su mayor fidelidad anatómica como por su facilidad para comparar con otras técnicas como la TC. Además es posible evaluar la intensidad de las lesiones a través de un método semicuantitativo como el propuesto $u$ otro de cuantificación más objetiva actualmente en estudio en nuestra unidad de medicina nuclear.

\section{Conclusión}

1) En niños con daño pulmonar crónico el SPECT de perfusión pulmonar muestra una alta proporción de estudios alterados y compromiso bilateral, especialmente en varones, con algunas características diferenciales según la etiología del cuadro.

2) Es posible graduar la intensidad de las alteraciones cintigráficas mediante un score semicuantitativo.

\section{Bibliografía}

1. Ciofetta G. Lung scintigraphy in the assessment of recurrent bronchitis and pneumonia in children. Q J Nucl Med 1997; 41:292-301.

2. Gordon I, Helms P, Fazio F. Clinical application of radionuclide lung scanning in infants and children. $\mathrm{Br} J$ Radiol 1981; 54:576-85.

3. Piepsz A, Wetzburger C, Spehl M y col. Critical evaluation of scintigraphy in cystic fibrosis: study of 113 patients. J Nucl Med 1980; 21:909-13.

4. Donnelly LF, Gelfand MJ, Brody AS, Wilmott RW. Comparison between morphologic changes seen on high-resolution $C T$ and regional pulmonary perfusion seen on SPECT in patients with cystic fibrosis. Pediatr Radiol 1997; 27:920-5.

5. Collart JP, Roelants V, Vanpee D y col. Is a lung perfusion scan obtained by using single photon emission computed tomography able to improve the radionuclide diagnosis of pulmonary embolism? Nucl Med Commun 2002; 23:1107-13.

6. Kwa SL, Theuws JC, van Herk M, Damen EM, Boersma $\mathrm{LJ}, \mathrm{Baas} \mathrm{P}$ et al. Automatic three-dimensional matching of CT-SPECT and CT-CT to localize lung damage after radiotherapy. J Nucl Med 1998;39:1074-80.

7. Similä S, Linna O, Lannig $P$ et al. Chronic lung damage caused by adenovirus 7: a ten years follow-up study. Chest 1981;80:127-31.

8. Becroft DM. Pulmonary sequelae of epidemic type 21 adenovirus infection: a 13-year follow-up. Arch Dis Child 1979;54:155-6.

9. Bhalla M, Turcios N, Aponte V, Jenkins M. Cystic fibrosis: scoring system with thin section CT. Radiology 1991;179:783-8.

10. Siegel MJ, Bhalla S, Gutierrez FR y col. Post-lung transplantation bronchiolitis obliterans syndrome: usefulness of expiratory thin-section CT for diagnosis. Radiology 2001; 220:445-62.

11. Soto G, Linares M, Díaz JC y col. Tomografía computada de alta resolución en niños con daño pulmonar por adenovirus: caracterización y correlación con función pulmonar. Rev Chil Radiol 2002;8:149-53. 